\title{
INCIDENCIA Y SIGNIFICACIÓN DE LA "PROLIFERACIÓN ACINAR ATIIPICA” EN LAS BIOPSIAS TRANSRECTALES DE PRÓSTATA
}

\author{
F. HERRANZ AMO, F. VERDÚ TARTAJO, J.M. DÍEZ CORDERO, D. SUBIRÁ RÍOS, \\ I. CASTAÑO GONZÁLEZ, M. MORALEJO GÁRATE, J.I. MARTÍNEZ SALAMANCA, \\ R. CABELLO BENAVENTE
}

Servicio de Urología. Hospital General Universitario Gregorio Marañón. Madrid.

Actas Urol Esp. 28 (6): 432-436, 2004

\section{RESUMEN \\ INCIDENCIA Y SIGNIFICACIÓN DE LA “PROLIFERACIÓN ACINAR ATÍPICA” EN LAS BIOPSIAS TRANSRECTALES DE PRÓSTATA}

OBJETIVO: Evaluar la incidencia de atipia acinar en nuestra serie, el porcentaje de cáncer de próstata y otras lesiones sospechosas o preneoplásicas en las biopsias sucesivas y establecer una estrategia de seguimiento en estos pacientes.

MATERIAL Y MÉTODO: Se han extraído de nuestra base de datos 117 pacientes con el diagnóstico de atipia acinar aislada o asociada a PIN de alto grado, 75 (64\%) fueron sometidos al menos a una segunda biopsia de la glándula. La edad, PSA, tacto rectal, volumen prostático, nódulo ecográfico y diagnóstico patológico previo se compararon con el resultado patológico de las sucesivas biopsias de la glándula.

RESULTADOS: La incidencia de atipia acinar fue del 4\%. El 46,7\% de los pacientes sometidos a rebiopsia presentaron cáncer de próstata. No encontramos diferencias significativas en las variables clínicas analizadas entre los pacientes con diagnóstico final de cáncer o no. Los pacientes con diagnóstico inicial de atipia acinar con PIN de alto grado asociado presentaron una mayor incidencia de cáncer en la rebiopsia que los pacientes con atipia acinar aislada ( $\mathrm{p}=0,007)$. El 94,3\% de las neoplasias diagnosticadas se detectaron en la $2^{-\underline{a}}$ y $3^{\text {a }}$ biopsia.

CONCLUSIONES: Los pacientes con atipia acinar tienen un riesgo elevado de tener un cáncer de próstata no detectado en la $1^{\text {a }}$ biopsia, por lo tanto deben de ser sometidos a rebiopsia en el menor plazo posible. La probabilidad de diagnosticar un cáncer después de una $3^{\text {a }}$ biopsia es muy pequeña.

PALABRAS CLAVE: Atipia acinar. ASAP. Cáncer de próstata. Neoplasia prostática intraepitelial.

\begin{abstract}
INCIDENCE AND SIGNIFICANCE OF “ATYPICAL ACINAR PROLIFERATION” IN TRANSRECTAL PROSTATIC BIOPSIES

OBJECTIVE: To determine the incidence of atypical acini in our series, the percentage of prostate cancer and other suspicious or preneoplastic lesions in the successive biopsies and to establish a follow up strategy in these patients.

MATERIAL AND METHOD: A total of 117 patients diagnosed with isolated atypical acini or associated with high grade PIN were obtained from our database, $75(64 \%)$ of these were submitted to at least a second biopsy of the gland. The age, PSA, digital rectal examination (DRE), prostate volume, ultrasound nodule and previous pathological diagnosis were compared with the pathology results of the successive biopsies of the gland.

RESULTS: Incidence of atypical acini was 4\%. A total of $46.7 \%$ of patients undergoing a repeat biopsy presented prostate cancer. We did not find significant differences in the clinical variables studied between patients with or without a definite cancer diagnosis. Patients initially diagnosed with acinar atypia with associated high grade PIN presented a higher incidence of cancer in the repeat biopsy than patients with isolated acinar atypia ( $\mathrm{p}=0.007)$. A total of $94.3 \%$ of all neoplasms diagnosed were detected in the $2^{\text {nd }}$ and $3^{\text {rd }}$ biopsy.

CONCLUSIONS: Patients with atypical acini are at high risk of having prostate cancer not detected in the first biopsy. They should, therefore, undergo a second biopsy as soon as possible. There is only a very small probability of detecting cancer after the $3^{\text {rd }}$ biopsy.
\end{abstract}

KEY WORDS: Atypical acini. ASAP. Prostate cancer. Intra-epithelial bladder neoplasm. 
$\mathrm{L}^{\mathrm{a}}$ a generalización del PSA en el estudio de los pacientes con síndrome prostático y su utilización, cada vez más frecuente, como diagnóstico precoz del cáncer de próstata, ha aumentado de forma muy importante el número de biopsias prostáticas realizadas. Además de un aumento considerable en la incidencia del cáncer de próstata, estamos observando el aumento de otros diagnósticos patológicos como neoplasia intraepitelial de alto grado y lo que los autores anglosajones definen como ASAP (atypical small acinar proliferation) o atipia acinar que nos plantean serias dudas sobre el manejo diagnóstico y seguimiento de estos pacientes.

El diagnóstico de adenocarcinoma de próstata se basa tanto en cambios citológicos como en su arquitectura. La presencia de anaplasia nuclear, nucleolo prominente, invasión estromal, pérdida de las células basales y cambios en la arquitectura son elementos imprescindibles para poder enunciar un diagnóstico de malignidad, la presencia de cristaloides o mucina basófila corrobora la presencia de malignidad ${ }^{1}$. Los focos de atipia acinar presentan algunos de los hallazgos patológicos pero no los suficientes para poder afirmar la existencia de un adenocarcinoma. Estos focos son generalmente pequeños y carecen del nucleolo prominente o carecen del suficiente número de otros hallazgos como: nucleomegalia, núcleo hipercromático, mucina basófila o cristaloides para poder emitir un diagnóstico ${ }^{1,2}$.

El significado clínico de esta atipia acinar es desconocido; mientras que algunos pacientes son diagnosticados finalmente de carcinoma, otros presentan una amplia variedad de lesiones proliferativas benignas que se asemejan al carcinoma. La incidencia de atipia acinar es muy variable entre el $0,5 \%$ y el 18\%3,4. En las sucesivas biopsias se diagnosticó cáncer entre el $42 \%$ y el 60\% de los casos ${ }^{5}$ y atipia acinar de nuevo entre el 5\% y el 9\% ${ }^{4}$.

El objetivo de este estudio es evaluar la incidencia de atipia acinar en nuestra serie, el porcentaje de cáncer de próstata y otras lesiones sospechosas o preneoplásicas en las biopsias sucesivas y establecer una estrategia de seguimiento en estos pacientes.

\section{MATERIAL Y MÉTODO}

Desde enero de 1995 hasta marzo de 2003 hemos realizado biopsia transrectal ecodirigida a
2.871 pacientes, a 521 pacientes se les practicó al menos una segunda biopsia. En el 4\% (117 pacientes) de los casos en el informe anatomopatológico se diagnosticó de atipia acinar. De estos pacientes 75 (64\%) tienen al menos una segunda biopsia y van a ser el objeto de este estudio.

Todos los pacientes realizaron preparación rectal mediante enemas de limpieza y profilaxis antibiótica oral. La ecografía transrectal se realizó con un ecógrafo Brüel \& Kjaer modelo 3535 con un transductor transrectal multiplanar a 7,5 Mhz. con canal de biopsia integrado tipo 8551, colocándose al paciente en posición de litotomía. El 96,5\% de las exploraciones fueron realizadas por el autor y el resto por un médico interno residente supervisado por el autor. La exploración se inició con la realización de un tacto rectal, tras la introducción del transductor se calculó el volumen de la glándula prostática según la formula del elipsoide (cálculo realizado por el software incluido en el ecógrafo). Se realizó biopsia transrectal de la glándula prostática con un sistema automático de punción y agujas calibre 18 $\mathrm{G}$, que extraen cilindros de $17 \mathrm{~mm}$ de longitud. Se realizaron 3 biopsias de la parte más lateral de cada lóbulo prostático intentando incluir en ellas la mayor cantidad de tejido de la zona periférica, incluyéndose en ellas los nódulos hipoecoicos. Las variables PSA, edad, volumen prostático y existencia de nódulo ecográfico se relacionaron con la existencia de cáncer en las sucesivas biopsias.

Se ha utilizado el programa informático SPSS 8.0 para el manejo estadístico de las variables. Las variables cualitativas se expresan en porcentajes de las mismas y las variables cuantitativas por la media, desviación típica, mínimomáximo y mediana. La comparación de variables continuas no paramétricas con variables cualitativas se ha realizado mediante el test Mann-Whitney.

\section{RESULTADOS}

La incidencia de cáncer de próstata global de la serie fue del 42,5\% (1.219 pacientes) (Tabla 1). En 117 casos (4\%) se diagnosticó atipia acinar y 75 pacientes (64\%) fueron sometidos al menos a una segunda biopsia, estos pacientes serán el objeto de este estudio. 
Tabla 1. Porcentaje de cáncer de próstata según las series de biopsias

\begin{tabular}{cccc}
\hline $\begin{array}{c}\text { Serie } \\
\text { de biopsia }\end{array}$ & Pacientes & Cáncer (\%) & $\begin{array}{c}\text { \% acumulado } \\
\text { de cáncer }\end{array}$ \\
\hline $\mathbf{1}^{\mathbf{a}}$ & 2.871 & $1.103(38,4 \%)$ & $90,5 \%$ \\
$\mathbf{2}^{\mathbf{a}}$ & 521 & $87(16,7 \%)$ & $97,6 \%$ \\
$\mathbf{3}^{\mathbf{a}}$ & 144 & $19(13,2 \%)$ & $99,2 \%$ \\
$\mathbf{4}^{\mathbf{a}}$ & 45 & $7(15,6 \%)$ & $99,8 \%$ \\
$\mathbf{5}^{\mathbf{a}}$ & 12 & $2(16,7 \%)$ & $99,9 \%$ \\
$\mathbf{6}^{\mathbf{a}}$ & 2 & 0 & $99,9 \%$ \\
$\mathbf{7}^{\mathbf{a}}$ & 1 & 0 & $99,9 \%$ \\
$\mathbf{8}^{\mathbf{a}}$ & 1 & $1(100 \%)$ & $100 \%$ \\
\hline
\end{tabular}

La edad media fue de 67,7 \pm 7,7 (45-83) años, la media de PSA fue de $11,3 \pm 7(2,9-37) \mathrm{ng} / \mathrm{ml}$, el $84 \%$ presentaban un tacto rectal no sospechoso, el $24 \%$ tenían un nódulo hipoecoico y el volumen medio de la glándula fue de 57,5 $\pm 29,6$ (18136) cc. En el informe patológico el $66,7 \%$ de los casos presentaban atipia acinar aislada, mientras que el 33,3\% restante tenían además PIN de alto grado. El 78,7\% de los casos de atipia acinar se detectó en la primera biopsia que se le realizó al paciente, el $17,3 \%$ en la $2^{\text {a }}$ biopsia, el $2,7 \%$ en la $3^{\text {a }}$ y el $1,3 \%$ en la $4^{\text {a }}$ biopsia. A partir de aquí consideraremos como primera biopsia la biopsia en la que se detectó la atipia acinar, independientemente de si fue o no la primera biopsia cronológica.

Al comparar los pacientes con atipia acinar que fueron sometidos a una segunda biopsia con los que no se sometieron a rebiopsia, observamos que los pacientes del estudio son más jóvenes $(\mathrm{p}=0,02)$, con un PSA más bajo $(\mathrm{p}=0,1)$, un volumen prostático menor $(\mathrm{p}=0,4)$, un porcentaje de tacto rectal sospechoso menor $(\mathrm{p}=0,1)$, menor porcentaje de nódulos hipoecoicos $(\mathrm{p}=0,5)$ y una asociación atipia acinar - PIN de alto grado mayor $(\mathrm{p}=0,6)$, existiendo sólo diferencias significativas en la edad (Tabla 2). El tiempo medio transcurrido entre la $1^{\mathrm{a}}$ y la $2^{\mathrm{a}}$ biopsia fue de $156 \pm 186$ (15990) días, el $50 \%$ de los pacientes se rebiopsió en menos de 3 meses y el 76\% en menos de 6 meses. No existiendo diferencias significativas $(p=0,5) \mathrm{si}$ el diagnóstico inicial fue de atipia acinar aislada (153 \pm 180 días) o de atipia acinar con PIN de alto grado (165 \pm 201 días).
Tabla 2. Relación entre los pacientes con atipia acinar incluidos

(al menos $2^{\text {a }}$ biopsia) y no incluidos (no $2^{\text {a }}$ biopsia) en el estudio

\begin{tabular}{lccc}
\hline Variable & Incluidos & Excluidos & P \\
\hline Edad & $67,7 \pm 7,7$ & $70,2 \pm 6,5$ & 0,02 \\
PSA & $11,3 \pm 7$ & $15,5 \pm 15,7$ & 0,1 \\
Volumen próstata & $57,5 \pm 29,6$ & $60 \pm 31,6$ & 0,4 \\
TR sospechoso & $16,2 \%$ & $26,8 \%$ & 0,1 \\
Nódulo ecográfico & $24,3 \%$ & $29,3 \%$ & 0,5 \\
AA+PIN-AG & $33,3 \%$ & $28,6 \%$ & 0,6 \\
\hline
\end{tabular}

TR: tacto rectal. AA+PIN-AG: atipia acinar y neoplasia intraepitelial de alto grado.

El diagnóstico final de la serie fue: cáncer en el 46,7\% (35/75) de los casos, tejido normal en el $32 \%$ (24/75), PIN de alto grado en el $14,6 \%$ $(11 / 75)$ y atipia acinar en el 6,7\% (5/75). Los resultados parciales obtenidos en las diferentes series de biopsias se detallan en la Figura 1. El $80 \%$ de los tumores se diagnosticó en la $2^{\mathrm{a}}$ biopsia, el $94,3 \%$ en la $3^{\text {a }}$ biopsia y el $97,1 \%$ en la $4^{\text {a }}$ biopsia.

No se encontró una relación significativa entre el diagnóstico final y la edad $(\mathrm{p}=0,6)$, el volumen prostático $(\mathrm{p}=0,2)$, el PSA $(\mathrm{p}=0,7)$, el tacto rectal $(\mathrm{p}=0,6)$ y el nódulo ecográfico hipoecoico $(\mathrm{p}=0,5)$ (Tabla 3).

Los pacientes que en la $1^{\text {a }}$ biopsia tenían atipia acinar y PIN de alto grado presentaron en el diagnóstico final una mayor incidencia de cáncer $\mathrm{y}$ de PIN de alto grado de forma significativa $(\mathrm{p}=0,007)$ (Tabla 4).

\section{DISCUSIÓN}

Aproximadamente en el 10\% de las biopsias de próstata, debido a alteraciones en el material remitido, no es posible distinguir entre lesiones proliferativas benignas o malignas. En 1993 se acuñó el término ASAP (atypical small acinar proliferation) ${ }^{9}$ para transmitir al clínico que no es posible efectuar un diagnóstico correcto y que debe de ser excluida la posibilidad de un carcinoma en ese paciente en concreto. En nuestro medio esto se refleja en los informes patológicos como "proliferación acinar atípica, atipia acinar o glandular" o más frecuentemente como "foco atípico sospechoso pero no diagnóstico de malignidad". 


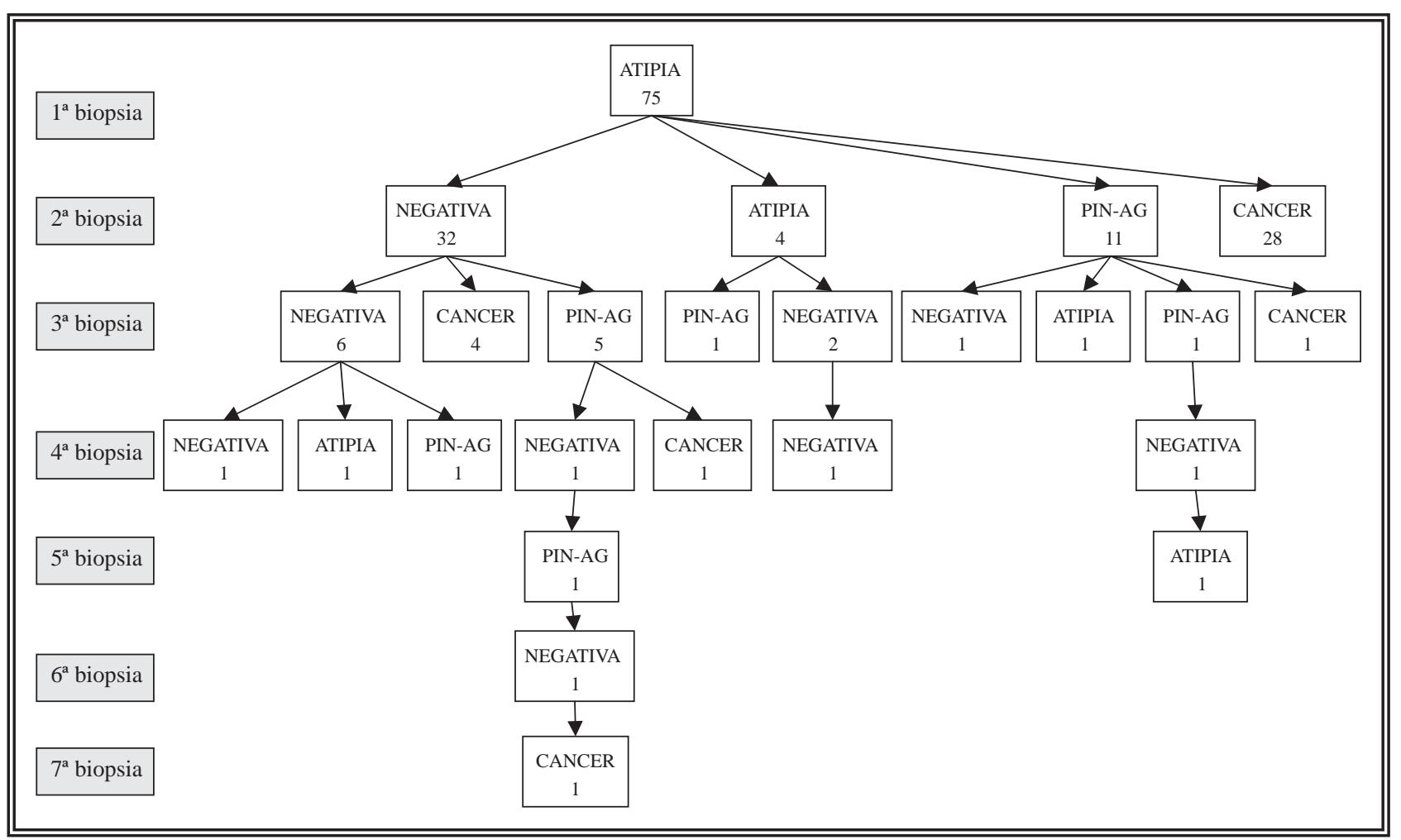

FIGURA 1. Resultado patológico de las diversas biopsias realizadas a los pacientes con atipia glandular.

Tabla 3. Relación entre el diagnóstico final y las variables estudiadas

\begin{tabular}{|c|c|c|c|c|c|c|c|}
\hline \multirow[t]{2}{*}{ Dx. final } & \multirow[t]{2}{*}{ Edad } & \multirow[t]{2}{*}{ PSA } & \multirow[t]{2}{*}{ Volumen } & \multicolumn{2}{|c|}{ Tacto rectal } & \multicolumn{2}{|c|}{$\begin{array}{c}\text { Nódulo } \\
\text { hipoecoico }\end{array}$} \\
\hline & & & & & & & $\begin{array}{l}\text { oico } \\
(-)\end{array}$ \\
\hline Negativo & $66,7 \pm 8,4$ & $11,5 \pm 6,5$ & $64,3 \pm 32,7$ & $16 \%$ & $84 \%$ & $28 \%$ & $72 \%$ \\
\hline Atipia acinar & $71,2 \pm 3,4$ & $16,5 \pm 6,4$ & $72,2 \pm 34,3$ & - & $100 \%$ & - & $100 \%$ \\
\hline PIN-AG & $64,9 \pm 7,6$ & $11,7 \pm 7,6$ & $54,5 \pm 30,2$ & $10 \%$ & $90 \%$ & $20 \%$ & $80 \%$ \\
\hline Cáncer & $68,8 \% \pm 7,6$ & $10,2 \pm 7,2$ & $51,5 \pm 26$ & $20 \%$ & $80 \%$ & $25,7 \%$ & $74,3 \%$ \\
\hline
\end{tabular}

PIN-AG: neoplasia intraepitelial prostática de alto grado.

En nuestra serie la incidencia de pacientes con atipia acinar fue del $4 \%$, similar a la incidencia publicada en la literatura que oscila entre el $1,5 \%$ y el $9 \%$ en series no seleccionadas ${ }^{3,4,6-8}$. La única diferencia o sesgo existente entre la población total de pacientes diagnosticados de atipia acinar y los incluidos en el estudio es la edad. Los pacientes incluidos en el estudio son más jóvenes, pero no existen diferencias significativas en cuanto a las otras variables analizadas en el estudio (PSA, tacto rectal, volumen prostático, nódulo ecográfico y coexistencia de PIN de alto grado). En nuestra serie el $46,7 \%$ y el 6,7\% de los pacientes presentaron cáncer o atipia acinar de nuevo al final del estudio. Estos datos coinciden con los comunicados en la literatura, cáncer de próstata entre el $40 \%$ y el $60 \%$ y de nuevo atipia acinar entre el $3,2 \%$ y el $9 \% 4,5,7,8$.

No hemos encontrado una diferencia significativa en las variables clínicas analizadas entre los pacientes con cáncer de próstata u otros diagnósticos al final del estudio, por lo tanto no podemos establecer factores de riesgo con los que indicar una $2^{\mathrm{a}}$ biopsia. Renshaw y cols. ${ }^{4}$ en su serie de 54 pacientes con ASAP y seguimiento con biopsias sucesivas, encuentran que los pacientes 
Tabla 4. Relación entre el hallazgo en la $1^{\text {a }}$ biopsia y el diagnóstico final

\begin{tabular}{lcc}
\hline & \multicolumn{2}{c}{ Primera biopsia } \\
\cline { 2 - 3 } $\begin{array}{l}\text { Diagnóstico } \\
\text { final }\end{array}$ & Atipia acinar & $\begin{array}{c}\text { Atipia acinar + } \\
\text { PIN-AG }\end{array}$ \\
\hline Negativo & $44 \%$ & $12 \%$ \\
Atipia acinar & $8 \%$ & $4 \%$ \\
PIN-AG & $6 \%$ & $28 \%$ \\
Cáncer & $42 \%$ & $56 \%$ \\
\hline
\end{tabular}

PIN-AG: neoplasia intraepitelial prostática de alto grado.

con diagnóstico final de cáncer son de mayor edad de forma significativa que el resto de los diagnósticos, pero no encuentran diferencias en el PSA ni en los hallazgos al tacto rectal. En la serie de 17 pacientes de Ouyang y cols. ${ }^{7}$ los pacientes con cáncer tenían un PSA mayor de forma significativa, al igual que en la serie de 55 pacientes de Borboroglu y cols. ${ }^{3}$.

El 94,3\% de las neoplasias diagnosticadas en nuestra serie se detectaron en la $2^{\mathrm{a}}$ y $3^{\mathrm{a}}$ biopsia, con la $4^{\text {a }}$ biopsia sólo se incrementó la detección de cáncer en un $2,8 \%$. Iczkowski y cols. ${ }^{5}$ diagnosticaron el $99 \%$ de las neoplasias en la $2^{\text {a }}$ y $3^{\text {a }}$ biopsia, concluyendo que después de un diagnóstico de ASAP no se deben de realizar más de 2 rebiopsias.

Para Iczkowski y cols. ${ }^{5}$ la atipia acinar no es una lesión neoplásica como lo sería el PIN de alto grado, sino que representa la muestra marginal de un cáncer existente en la glándula. En cambio, otros autores ${ }^{7}$ consideran que no es una entidad diagnóstica única, sino que engloba una serie de lesiones no relacionadas que van a presentar un curso clínico impredecible.

En resumen, la probabilidad de diagnosticar una neoplasia después de un diagnóstico de atipia acinar o ASAP es muy elevada, por lo tanto se debería de repetir la biopsia de la glándula en el menor plazo posible. La realización de una $4^{\text {a }}$ biopsia en estos pacientes se debería de reservar para pacientes jóvenes y con factores pronósticos clínicos y patológicos muy desfavorables, ya que la probabilidad de diagnosticar un cáncer es muy baja.

\section{REFERENCIAS}

1. EPSTEIN JI.: Diagnostic criteria of limited adenocarcinoma of the prostate on needle biopsy. Human Pathol 1995; 26: 223-229.

2. MOSTOFI FK, SESTERHENN IA, DAVIS CJ Jr.: Prostatic carcinoma: problems in the interpretation of prostatic biopsies. Hum Pathol 1992; 23: 223-241.

3. BORBOROGLU PG, SUR RL, ROBERTS JL, AMLING CL.: Repeat biopsy strategy in patients with atypical small acinar proliferation or high grade prostatic intraepithelial neoplasia on initial prostate needle biopsy. J Urol 2001; 166: 866-870.

4. RENSHAW AA, SANTIS WF, RICHIE JP.: Clinicopathological characteristics of prostatic adenocarcinoma in men with atypical prostate needle biopsies. J Urol 1998; 159: 2018-2021.

5. ICZKOWSKI KA, BASSLER TJ, SCHWOB VS et al.: Diagnosis of "supicious for malignancy" in prostate biopsies: predictive value for cancer. Urology 1998; 51: 749-758.

6. CHEVILLE JC, REZNICEK MJ, BOSTWICK DG.: The focus of "atypical glands, suspicious for malignancy" in prostatic needle biopsy specimens: incidence, histologic features, and clinical follow-up of cases diagnosed in a community practice. Am $J$ Clin Pathol 1997; 108: 633-640.

7. OUYANG RC, KENWRIGHT DN, NACEY JN, DELAHUNT B.: The presence of atypical small acinar proliferation in prostate needle biopsy is predictive of carcinoma on subsequent biopsy. BJU International 2001; 87: 70-74.

8. ICZKOWSKI KA, CHEN HM, YANG XJ, BEACH R.: Prostate cancer diagnosed after initial biopsy with atypical small acinar proliferation suspicious for malignancy is similar to cancer found on initial biopsy. Urology; 60: 851-854.

9. BOSTWICK DG, SRIGLEY J, GRIGNON D et al.: Atypical adenomatous hyperplasia of the prostate: morphologic criteria for its distinction from well-differentiated carcinoma. Hum Pathol 1993; 24: 819832 .

Dr. F. Herranz Amo

C/ Doctor Esquerdo, $155^{\underline{a}}-7^{\circ} 3$

28007 Madrid

(Trabajo recibido el 23 junio de 2003) 